\title{
ACUTE KIDNEY INJURY
}

NEW ANTAGONIST PREVENTS I/R INJURY

Pharmacologic antagonism of the mineralocorticoid receptor is effective in preventing acute kidney injury (AKI) after ischaemia/reperfusion (I/R), but the underlying mechanisms and the renal cell types targeted by mineralocorticoid receptor antagonists (MRAs) were previously unknown. Now, research by Frédéric Jaisser and colleagues shows that BR-4628 (a novel non-steroidal MRA) protects against oxidant damage induced by renal I/R, and that its beneficial effects can be blocked by a selective endothelin $B$ receptor $\left(\mathrm{ET}_{\mathrm{B}}\right)$ antagonist.

The researchers administered BR-4628 to a rat model of transient bilateral renal $\mathrm{I} / \mathrm{R}$ and examined the effects on renal injury and the endothelin pathway, as prepro-endothelin-1 is upregulated following I/R-induced AKI. Firstly, the researchers found that administration of BR-4628 before or $3 \mathrm{~h}$ after $\mathrm{l} / \mathrm{R}$ was efficient in preventing renal injury and in improving renal function, as verified by histologic assessment and molecular analysis of markers of apoptosis, inflammation, and tubular injury. Secondly, the researchers noted that $\mathrm{I} / \mathrm{R}$ induced inactivation of $\mathrm{ET}_{\mathrm{B}}$ in their model by sulfenic acid modification, which consequently decreased eNOS activity and nitric oxide production. Consequently, rats co-administered BR-4628 and an $\mathrm{ET}_{\mathrm{B}}$ antagonist maintained elevated creatinine levels and exhibited histologic indicators of renal injury, thus supporting a direct action of BR-4628 on $\mathrm{ET}_{\mathrm{B}}$ activation.

"Using genetic mouse models we plan to identify which cell type is involved in the beneficial effects of MRAs in renal I/R injury, focusing on the endothelial and vascular smooth muscle cells that we previously reported to be key targets of mineralocorticoids for vascular function," explains Jaisser. "Moreover, we are performing translational research using the large white pig in order to be closer to the human setting and reinforce the rationale for future clinical trials in AKI related to impaired renal perfusion." The researchers have also initiated a randomized interventional clinical trial (EPURE Transplant; NCT02490904) to test the potential efficacy of eplerenone, another MRA, in kidney transplantation.

Jessica K. Edwards

Original article Barrera-Chimal, J. et al. Sulfenic acid moficiation of endothelin $B$ receptor is responsible for the benefit of a nonsteroidal mineralocorticoid receptor antagonist in renal ischemia. J. Am. Soc. Nephrol. doi:10.1681/ASN.2014121216 